Chromatin immunoprecipitation (ChIP) assay was used to screen and verify the GCLM target genes.

Results We found that glucose deprivation condition upregulated the expression of GCLM rather than hypoxia or $\mathrm{H} 2 \mathrm{O} 2$. Compared to adjacent tumor tissues, the expression of GCLM was upregulated in CRC tumor tissues. The high expression of GCLM predicted poor prognosis in CRC patients. Inhibition of GCLM results in decreased proliferation rate, migration and invasion ability and sphere formation in HCT116 and DLD1 in vitro. Similarly, GCLM inhibition suppressed CRC tumorigenesis and metastasis in xenograft models and PDX models in vivo. The cancer cell stem cell markers were also downregulated after GCLM inhibition. Interestingly, we found that glucose starvation could rescue the anti-cancer phenotypes of GCLM inhibition and led to nucleus-translocation and accumulation of GCLM. ChIP assay showed GCLM could interact with octamer-binding transcription factor 4 (OCT4) promoter, a cancer stem cell marker, to increase its expression, and the target gene of OCT4 showed a positive correlation with GCLM expression in CRC patients.

Conclusions Our data showed that GCLM displayed nucleus accumulation in the condition of glucose deprivation which increased the transcription of OCT4. This study revealed an unorthodox oncogenic function of GCLM in colorectal carcinoma and suggested GCLM as a potential therapeutic target in CRC.

\section{IDDF2019-ABS-0293 A MICROBIOME PILOT STUDY: THE EXPLORATION OF THE GUT-SKIN AXIS IN HIDRADENITIS SUPPURATIVA}

\begin{abstract}
${ }^{1}$ Suk Yee Lam*, ${ }^{2}$ Djawad Radjabzadeh, ${ }^{1}$ Hester Eppinga, ${ }^{3}$ Hessel $\mathrm{H}$ van der Zee, ${ }^{2}$ Robert Kraaij, ${ }^{1}$ Sergey R Konstantinov, ${ }^{1}$ Gwenny M Fuhler, ${ }^{3}$ Errol P Prens, ${ }^{3}$ Hok Bing Thio, ${ }^{1}$ Maikel P Peppelenbosch. 'Department of Gastroenterology and Hepatology, Erasmus University Medical Center, Netherlands; ${ }^{2}$ Department of Internal Medicine, Erasmus University Medical Center, Netherlands; ${ }^{3}$ Department of Dermatology, Erasmus University Medical Center, Netherlands
\end{abstract}

\subsection{6/gutjnl-2019-IDDFabstracts.63}

Background The concurrent occurrence of gut and skin diseases has led to the postulation of the existence of a gut-skin axis in which gut microbiota is thought to contribute to extra-intestinal skin manifestations. A dysbiotic skin microbiome has been demonstrated in the chronic inflammatory skin disease hidradenitis suppurativa (HS), but it remains unknown whether microbial disturbances of the gut are engaged in the complex pathogenesis. This study investigates the fecal microbiome in HS patients and healthy controls and explores the possibility of a gut-skin axis in HS.

Methods A total of 17 HS patients and 20 healthy controls provided a stool sample for $16 \mathrm{~S}$ ribosomal RNA (rRNA) gene sequencing. A subset of axillary skin samples was available for concurrent microbial analysis of HS lesions $(n=6)$, paired nonlesions $(n=3)$ and healthy skin of controls $(n=7)$. No oral antibiotics and no topical antibiotics/steroids were used in respectively eight weeks and seven days prior to sample collection.

Results The majority of 16S rRNA gene sequences at both the fecal and axillary skin sites were assigned to phyla Bacteroidetes, Firmicutes, Proteobacteria and Actinobacteria. Based on the overall bacterial community, two distinct clusters could be distinguished between the gut and the skin using principal coordinates analysis (PCoA). Furthermore, significant differences in bacterial composition were observed in fecal samples of HS patients when compared to healthy controls. However, we did not detect altered microbial signatures in the skin samples taken from HS lesions, non-lesions, and healthy controls.

Conclusions These findings indicate the presence of a distinct fecal microbiome in HS patients compared to healthy controls, suggesting the involvement of a gut-skin axis in the etiology of HS. Dysbiosis of the cutaneous microbiome in HS could not be confirmed here, but larger studies are warranted to further elucidate the role of microbiota as a potential part of the gut-skin axis in HS.

\section{IDDF2019-ABS-0294 HSF2 PROMOTE THE MUCOSAL REPAIR IN ULCERATIVE COLITIS BY INHIBITING PRO- INFLAMMATORY CYTOKINE AND PROMOTING TGF- $\beta$ EXPRESSION}

JunKun Niu*. Department of Gastroenterology, The First Affiliated Hospital of Kunming Medical University, China

\subsection{6/gutjnl-2019-IDDFabstracts.64}

Background In our previous study, we found that fecal heat shock transcription factor 2 (HSF2) concentration may be an evaluation index for predicting the mucosal healing of Ulcerative Colitis (UC). So this study is aiming to explore the mechanism of HSF2 in the mucosal repair of UC.

Methods The DAI, colon damage score and histopathology score were recorded in DSS induced colitis. The transcription and expression level of HSF2 and TGF- $\beta$ in the mice colon tissue were detected by PCR and immunohistochemistry. HSF2 levels in HT-29 cells was manipulated by RNA interference and plasmids transfection. The concentrations of IL-1 $\beta$, TNF$\alpha$ and TGF- $\beta$ in cells supernatant were detected by ELISA after induced by LPS. The phosphorylation level of ERK, P38, JNK and $S$ mad2/3 were detected by Western Blot.

Results The DAI, colon damage and pathology score of methylprednisolone(MP)treatment group were lower than DSS model group (figure 1). The transcription and expression of HSF 2 and TGF- $\beta$ in colon mucosal tissue were increased and decreased synchronously in DSS model group and MP treatment group(figure 2). The phosphorylation level of ERK, P38 and JNK increased significantly in HSF2 siRNA compared with the negative control group. The concentrations of IL-1 $\beta$ and TNF- $\alpha$ in the supernatant of HT-29 cells increased in HSF2 siRNA group. On the contrary, the phosphorylation level of ERK, P38 and JNK decreased significantly in HSF2FLAG plasmid transfection compared with the Blank Vector group. The concentrations of IL- $1 \beta$ and TNF- $\alpha$ in the supernatant of HT-29 cells reduced (figure 3 , figure 4 ).

The phosphorylation level of Smad2/3 decreased in HSF2 siRNA compared with the negative control group. The concentrations of TGF- $\beta$ in the supernatant of HT-29 cells reduced in HSF2 siRNA group. On the contrary, the phosphorylation level of Smad2/3 increased significantly in HSF2FLAG plasmid transfection compared with the Blank Vector group. The concentrations of TGF- $\beta$ in the supernatant of HT-29 cells increased (Figure 4).

Conclusions HSF2 can promote the mucosal repair of ulcerative colitis by inhibiting the inflammatory response and promoting mucosal repair factor expression. 